\title{
Commentary: Zebras and horses: Is a thorough history and physical important in the age of advanced imaging?
}

\author{
Riyad Karmy-Jones, MD, FACS, FRCSC
}

\author{
From Thoracic/Vascular/Trauma Surgery, Peacehealth Southwest Medical Center, Vancouver, Wash. \\ Disclosures: Author has nothing to disclose with regard to commercial support. \\ Received for publication April 18, 2019; revisions received April 18, 2019; accepted for publication April 18, \\ 2019; available ahead of print June 20, 2019. \\ Address for reprints: Riyad Karmy-Jones, MD, FACS, FRCSC, 505 NE 87th Ave, Bldg B, Suite 301, Vancouver, \\ WA 98664 (E-mail: rkarmy-jones@ peaecehealth.org). \\ J Thorac Cardiovasc Surg 2020;159:e327-8 \\ 0022-5223/ $\$ 36.00$ \\ Copyright (C) 2019 by The American Association for Thoracic Surgery \\ https://doi.org/10.1016/j.jtcvs.2019.04.092
}

Pichert and colleagues ${ }^{1}$ describe a rare scenario that presented technical challenges to an already difficult case. Apart from the technical considerations, the authors present the case as an illustration of the value of the history and physical examination.

The value of the history and physical examination is generally assumed, but not often tested. Anecdotally, all of us can attest to cases where it has led to diagnosis or critical change in management, as well as cases for whom an inadequate history and physical led to potential problems. This is a regular issue in both outpatient and emergency settings. Currently, many surgeons find that we are getting referrals with inadequate pre-evaluation, putting the onus on us to do a complete assessment of the patient. Although not well studied, at least in our region after discussion with many colleagues, there are 2 reasons. The first is that primary care physicians are under increasing pressure to "get through the day" with brief appointments and therefore refer patients (often "urgently") to surgeons simply to get the patient out of the office. In addition, medical legal concerns seem to prevent primary care physicians from assuming any possibility of taking responsibility for some potentially surgical decision making. In the acute care setting, there is such dependency on advanced imaging that taking a history and performing a physical examination often take a secondary role. There is rational for this, for example in the trauma population in whom it has been shown that imaging reduces the incidence of missed injuries, as well as surgical planning (as the authors note), but again there is such pressure to rely on imaging rather than a comprehensive clinical assessment that important issues get missed (eg, is the patient do not resuscitate).

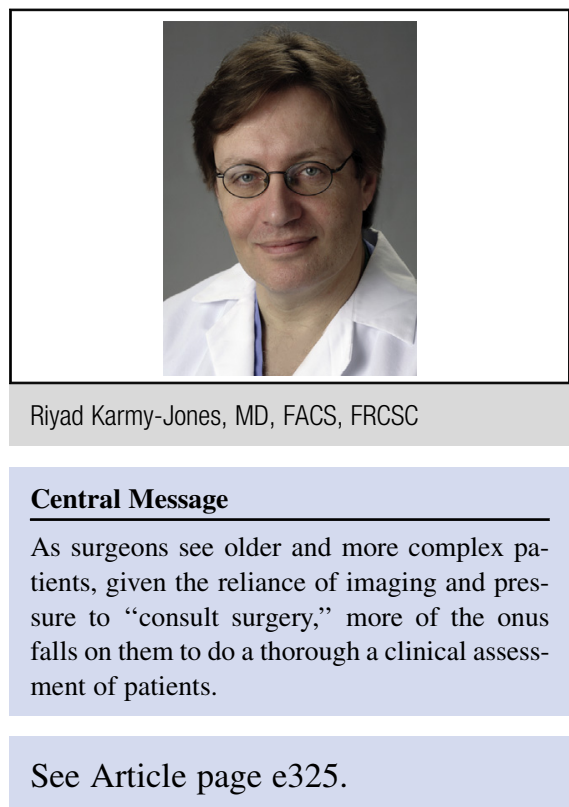

There are not much data on correlating physical examination and history with outcomes, but where there is resides largely in medical literature. Articles have suggested that our internal medicine colleagues think that a proper history and physical are valuable, that there is variable confidence across in the process across a broad spectrum of clinical experience, that there is fear that the "art" is being lost, and that there is a push to enhance training at all levels, even using simulation for specific portions of the examination.

In this case, the examination would have demonstrated at least a cervical and abdominal scar. I am presuming that the patient received a computed tomography angiography (appropriately). With a history of lye ingestion, there can be upper airway issues, so an important question is evidence of upper airway issues that might make anesthetic management more difficult. Typically, the colon tends to move to the right side of the chest, but there can be issues such as dilation, torsion, and stricture. An important question to answer is how has the conduit performed? Was the right or left colon used?

In the end, Pichert and colleagues ${ }^{1}$ handled a difficult case with skill. It could be argued that the computed tomography scan showed all that was needed to proceed. 
However, as the authors describe, it is often the history and physical examination that separate the horses from the zebras.

\section{Reference}

1. Pichert M, Mori M, Mangi A. Sternotomy for aortic dissection with substernal pull through: the importance of surgical history. J Thorac Cardiovasc Surg. 2020;159: e325-6. 